Bundesgesundheitsbl $2020 \cdot 63: 761-770$ https://doi.org/10.1007/s00103-020-03151-6 Online publiziert: 18. Mai 2020

(c) Der/die Autor(en) 2020

Holger Storf $f^{1,2} \cdot$ Jürgen Stausberg ${ }^{3} \cdot$ Gerhard Kindle $^{4,5} \cdot$ Bernd Quadder $^{6,11}$. Miriam Schlangen ${ }^{7} \cdot$ Maggie C. Walter $^{8} \cdot$ Frank Ückert $^{9} \cdot$ Thomas O. F. Wagner $^{10} \cdot$ NAMSE-Register-Strategiegruppe

${ }^{1}$ Medical Informatics Group (MIG), Universitätsklinikum Frankfurt, Frankfurt am Main, Deutschland; ${ }^{2}$ Datenintegrationszentrum (DIZ), Dezernat 7 - Informations- und Kommunikationstechnologie (DICT), Universitätsklinikum Frankfurt, Frankfurt am Main, Deutschland; ${ }^{3}$ Institut für Medizinische Informatik, Biometrie und Epidemiologie (IMIBE), Universitätsklinikum Essen, Essen, Deutschland; ${ }^{4}$ Institut für Immundefizienz, Centrum für Chronische Immundefizienz (CCI), Universitätsklinikum Freiburg, Medizinische Fakultät, Universität Freiburg, Freiburg, Deutschland; ${ }^{5}$ Zentrum für Biobanking, FREEZEBiobank, Universitätsklinikum Freiburg, Medizinische Fakultät, Universität Freiburg, Freiburg, Deutschland; ${ }^{6}$ Allianz Chronischer Seltener Erkrankungen ACHSE e. V., Berlin, Deutschland; $7{ }^{7}$ Geschäftsstelle des Nationalen Aktionsbündnisses für Menschen mit Seltenen Erkrankungen, Bonn, Deutschland; ${ }^{8}$ FriedrichBaur-Institut, Neurologische Klinik und Poliklinik, Ludwig-Maximilians-Universität München, München, Deutschland; ${ }^{9}$ Medizinische Informatik in der Translationalen Onkologie, Deutsches Krebsforschungszentrum, Heidelberg, Deutschland; ${ }^{10}$ Frankfurter Referenzzentrum für Seltene Erkrankungen (FRZSE), Universitätsklinikum Frankfurt, Frankfurt am Main, Deutschland; " Deutsche Sarkoidose-Vereinigung e. V., Meerbusch, Deutschland

\title{
Patientenregister für Seltene Erkrankungen in Deutschland: Konzeptpapier der Strategiegruppe „Register" des Nationalen Aktionsbündnisses für Menschen mit Seltenen Erkrankungen (NAMSE)
}

Wege aufzeigen, um aus den erhobenen Daten den größtmöglichen Nutzen ziehen zu können.

Es wird dabei nicht angestrebt, die kreative Kraft der einzelnen Akteure bei Planung, Einrichtung oder Betrieb durch übertriebene formale Vorgaben einzuschränken. Es ist vielmehr Absicht, aktuellen oder potenziellen Interessenten Hinweise zu geben, worauf bei Planung, Einrichtung und Betrieb eines Registers $\mathrm{zu}$ achten ist. Selbst wenn das Register nur einen zeitlich und thematisch fokussierten Zweck erfüllen soll, ist es sinnvoll, zu prüfen, ob die erhobenen Daten durch die Zusammenschau mit anderen Datensammlungen an Wert gewinnen und auch über die eigentlich geplante Nutzungsdauer hinaus für Forschungszwecke wichtige Dienste leisten können. Frühzeitige Klärung kann den späteren
Aufwand durch Vermeidung einer nachträglichen Einholung von Einwilligungserklärungen reduzieren und vom Start weg die Motivation bei Patienten und Versorgern verbessern.

\section{Sachstand}

Nationaler Aktionsplan: Umsetzung der Maßnahmen aus dem Handlungsfeld Register

Der Nationale Aktionsplan für Menschen mit Seltenen Erkrankungen [1] enthält 52 konkrete Maßnahmen, u.a. in den Handlungsfeldern Versorgung, Forschung, Diagnose und Informationsmanagement. Maßnahmenvorschlag 27 sieht vor, ein zentrales, nationales Verzeichnis bestehender Register aufzubauen. Die Entwicklung eines solchen
Stand von Registern auf dem Gebiet der
Seltenen Erkrankungen skizzieren und 


\section{Infobox 1 Übersicht na- \\ tionaler und europäischer (Forschungs-)Projekte mit Thema Register}

- RD-CONNECT (http://www.rd-connect.eu) Entwicklung einer modellhaften weltweiten Registerplattform für SE am Beispiel der neuromuskulären Erkrankungen (DG Research)

- PARENT-JA (http://www.patientregistries. eu)

Unterstützung der EU-Mitgliedsstaaten bei der Entwicklung von Registern für SE und Non-SE; Ziel: Harmonisierung der Entwicklung und Steuerung von Registern zur Ermöglichung von Sekundärdatenanalyse für Public Health und Forschung (DG SANCO)

- GRDR (Global Rare Diseases Patient Registry and Data Repository; http://www. grdr.info)

Pilotprogramm für ein weltweites krankheitsübergreifendes Register zu SE, das am amerikanischen National Institute of Health angesiedelt ist

- EXPAND - Expanding Health Data Interoperability Services (http://www. expandproject.eu/)

- OSSE - Open Source Registry System for Rare Diseases (http://www.osse-register. de/)

- TMF - Technologie- und Methodenplattform für die vernetzte medizinische Forschung e. V.; verschiedene Leitfäden u. a. zu Datenqualität und -schutz (http:// www.tmf-ev.de/)

- Deutsches Netzwerk Versorgungsforschung (DNVF; http://www.netzwerkversorgungsforschung.de/)

- Europäische Referenznetzwerke (ERN) Die Europäische Kommission hat unter den Europäischen Referenznetzwerken (ERN) eine Reihe von Projekten zu Registern für Seltene Erkrankungen zur Förderung beschlossen, die mit ihrer Arbeit begonnen haben (z. B. ERN-RITA, Metab-ERN, ERN-LUNG, ERN-EURO-NMD)

Webportals, einer Art „Telefonbuch für Register" sollte in der Strategiegruppe Register (siehe Maßnahmenvorschlag 28) thematisiert werden. Die Entwicklungen diesbezüglich wurden mit zentralen europäischen Aktivitäten abgestimmt, um Parallelstrukturen zu vermeiden. Da in der Zwischenzeit erkennbar wurde, dass die European Platform on Rare Disease Registration (EU RD Platform [2]) eine European Rare Disease Registry Infrastructure (ERDRI) entwickelt, die sowohl ein zentrales Metadatenregister (Central Metadata Repository; ERDRI.mdr) als auch ein Verzeichnis der SE-Register (European Directory of Registries; ERDRI.dor) enthält, wurde eine eigene nationale Entwicklung nicht vorangetrieben.

Mit dem Ziel, langfristig die Qualität und Interoperabilität von nationalen Registern zu erhöhen, sieht Maßnahmenvorschlag 28 die Etablierung einer Strategiegruppe „Register für Seltene Erkrankungen" vor. Diese soll Entwicklungen auf nationaler und internationaler Ebene berücksichtigen, um Empfehlungen für nationale Initiativen zu entwickeln. Hierzu wurde das vorliegende Konzeptpapier durch die Verantwortlichen erstellt. Primäres Ziel ist die Etablierung der Strategiegruppe als Expertengremium und Kommunikationsplattform für bestehende und neue Register und als nationaler Ansprechpartner für Anfragen aus anderen Ländern (z.B. Europäische Kommission, International Rare Diseases Research Consortium). Ein weiterer Arbeitsauftrag sind die Konsentierung und Implementierung sowie mit der Zeit ggf. die Anpassung eines Minimaldatensatzes zur Verwendung in Registern für Seltene Erkrankungen sowie die Abbildung der verwendeten Datenelemente bzw. Datenschemata in einem Metadata Repository. Dieses Verzeichnis soll Elemente enthalten, die in verschiedenen Registern abgefragt werden können und so der Interoperabilität dienen. Die Strategiegruppe hat 2016 ihre Arbeit aufgenommen.

Das Projekt „OSSE - Open SourceRegistersystem für Seltene Erkrankungen " ist ein wesentlicher Bestandteil bei der Umsetzung der Maßnahmen aus dem Handlungsfeld Register, insbesondere für Maßnahmenvorschlag 29, der Entwicklung eines Werkzeugkastens für krankheitsspezifische Register für Seltene Erkrankungen. OSSE stellt Patientenorganisationen, Ärzten, Wissenschaftlern und anderen Nutzern eine Open-Source-Software zur Verfügung, um ein eigenes Patientenregister zu entwickeln oder bereits bestehende Register mittels „Brückenkopf“ an eine Registerschnittstelle anzubinden. Dadurch wird die deutsche Registerlandschaft verbessert und entspricht dann den europäischen Richtlinien bezüglich Minimaldatensatz, Datenqualität und anderen Anforderungen (siehe European Union Committee of Experts on Rare Diseases (EUCERD) Recommendation on RD Registries [3]). Gleichzeitig wird die notwendige Interoperabilität erreicht, die das Föderieren solcher Register auf nationaler und internationaler Ebene erlaubt, z. B. mittels dezentraler Suchen, die dem Datenschutz entsprechen [4]. Seit Anfang 2015 ist die erste verfügbare Version der OSSE-Registersoftware auf der Webseite www.osse-register.de kostenlos herunterzuladen. OSSE-Register sind von vornherein auf Interoperabilität ausgelegt und können zentral genutzt werden oder auf nationaler oder internationaler Ebene föderiert werden. Beispielsweise sind verteilte Suchen über mehrere Instanzen hinweg möglich, die den Anforderungen an den Datenschutz entsprechen und dabei die Datenhoheit weiterhin gewährleisten [5]. Ebenfalls wird die Trennung von identifizierenden und medizinischen Daten durch die Einbindung eines ID-Managementdienstes, wie z.B. der Mainzelliste [6], unterstützt. Im Kontext der europäischen Referenznetzwerke für Seltene Erkrankungen (ERNs) wird partiell ebenfalls auf OSSE zurückgegriffen. Das Konzept und die Umsetzung von OSSE wurden 2017 im Bundesgesundheitsblatt publiziert [7]. $\mathrm{Zu}$ weiterführenden Informationen wird entsprechend hierauf verwiesen.

Für Seltene Erkrankungen, die so „selten“ sind, dass ein eigenes Register nicht sinnvoll erscheint, soll ein nichtkrankheitsspezifisches Register entwickelt werden. Vorgesehen ist, dass dieses Register auf der Technik des Modellregisters OSSE aufbaut. Verantwortlich für die Umsetzung ist die zuvor genannte Strategiegruppe "Register" (Maßnahmenvorschlag 32).

Ein Workshop zur Sammlung und Klärung offener Fragen hinsichtlich eines Registers für unklare Diagnosen (Maßnahmenvorschlag 30) hat stattgefunden. Die Ergebnisse des Workshops wurden aufgegriffen und in der Strategiegruppe Register berücksichtigt. Parallel ist im Sommer 2015 das vom Bundesministerium für Bildung und Forschung 
(BMBF) geförderte Projekt „VarWatch“ unter Federführung der Christian-Albrechts-Universität zu Kiel und mit Unterstützung der Arbeitsgruppe „Molekulare Medizin“ der Technologie- und Methodenplattform für die vernetzte medizinische Forschung e.V. (TMF) an den Start gegangen. Mit VarWatch ist der Aufbau einer Datenbank für genetische Varianten geplant, die bei Patienten mit syndromalen Störungen unklarer Ätiologie gefunden werden. In den anschließenden 2 Jahren wurde daran gearbeitet, VarWatch technisch zu etablieren, bei potenziellen Nutzern bekannt zu machen und ein Konzept für den langfristigen Betrieb zu entwickeln (Maßnahmenvorschlag 31).

\section{Bestandsaufnahme zu SE- Registern national und europaweit}

Aktuelle Informationen über Inhalt und Qualität von SE-Registern liegen nur sehr unvollständig vor. Daher wurde durch die NAMSE-Geschäftsstelle eine internetbasierte Befragung zur nationalen Situation durchgeführt. Die Teilnehmer beantworteten Fragen u.a. in den Bereichen Erkrankungsverlauf, Datenqualität, Technik, Finanzierung und Vernetzung mit anderen Registern. Zeitgleich fand eine europaweite Befragung ähnlichen Inhalts im Rahmen des EU-Projektes EPIRARE statt [8].

Obwohl die Ergebnisse bereits aus dem Jahr 2013 stammen, kann davon ausgegangen werden, dass sich die Situation nicht grundlegend geändert hat. Zusammenfassend ergab sich für Deutschland ein ausgeprägter Verbesserungsbedarf zu ganz grundlegenden Fragen, wie beispielsweise Datenstruktur (z.B. keine Codierung nach ICD-10 oder OrphaNummer), Datenschutz und Technik. Die Mehrzahl der Register wurde aus Mitteln zeitlich befristeter Forschungsprojekte finanziert. Perspektiven zur langfristigen Finanzierung aus anderen Quellen wurden bisher nicht entwickelt. Es wurde deutlich, dass eine öffentlich zugängliche Informationsquelle mit Dokumenten, konkreten Empfehlungen und Problemlösungen sowie eine geeignete Software für krankheitsspezifische SE-Register fehlen.

Bundesgesundheitsbl 2020 63:761-770 https://doi.org/10.1007/s00103-020-03151-6 (c) Der/die Autor(en) 2020

H. Storf · J. Stausberg · G. Kindle · B. Quadder · M. Schlangen · M. C. Walter · F. Ückert · T. O. F. Wagner $\cdot$ NAMSE-Register-Strategiegruppe

\section{Patientenregister für Seltene Erkrankungen in Deutschland: Konzeptpapier der Strategiegruppe „Register" des Nationalen Aktionsbündnisses für Menschen mit Seltenen Erkrankungen (NAMSE)}

\section{Zusammenfassung}

Der Nationale Aktionsplan für Menschen mit Seltenen Erkrankungen (SE) enthält 52 konkrete Maßnahmen, u. a. in den Handlungsfeldern Versorgung, Forschung, Diagnose und Informationsmanagement. Mit dem Ziel, langfristig die Qualität und Interoperabilität von nationalen Registern zu erhöhen, sieht Maßnahmenvorschlag 28 die Etablierung einer Strategiegruppe „Register für Seltene Erkrankungen“ vor. Diese Strategiegruppe hat 2016 ihre Arbeit aufgenommen. Sie berichtet hier über Entwicklungen auf nationaler und internationaler Ebene, um Empfehlungen für nationale Initiativen daraus abzuleiten. Zusätzlich werden die Konsentierung und Implementierung sowie mit der Zeit ggf. die Anpassung eines Minimaldatensatzes zur Verwendung in Registern für Seltene Erkrankungen erläutert. Zusätzlich werden die verwendeten Datenelemente bzw. -schemata in einem sog. Metadata Repository abgebildet. Dieses Positionspapier wurde durch die Strategiegruppe sowie weitere Autoren erarbeitet und innerhalb der Gruppe konsentiert. Es wird als Konzeptpapier zum Aufbau und Betrieb von Registern der Strategiegruppe „Register" veröffentlicht.

Schlüsselwörter

Register - Seltene Erkrankungen .

Konzeptpapier · Datenschutz

\section{Patient registries for rare diseases in Germany: concept paper of the NAMSE strategy group}

\section{Abstract}

The National Action Plan for People with Rare Diseases contains 52 concrete actions, including in the fields of care, research, diagnosis, and information management. With the aim of improving the quality and interoperability of national registries in the long term, action 28 proposed the establishment of a "Rare Diseases Registry" strategy group. The strategy group began its work in 2016. In this report, the group takes into account developments at the national and international level in order to develop recommendations for national initiatives. In addition to this, the group reports on consent and implementation as well as on the adaptation of a minimal dataset for use in rare disease registries and mapping the used data elements and schemata in a metadata repository. This position paper was created by the strategy group together with additional authors. The paper reached a consensus within the strategy group and can be seen as a concept paper of the Rare Diseases Registry strategy group.

\section{Keywords}

Registries - Rare diseases - Concept paper . Data protection

\section{Unterstützende europäische Aktivitäten zu SE-Registern}

Speziell im Bereich der SE mit national teilweise sehr geringen Patientenzahlen bei oftmals fehlenden evidenzbasierten Therapieoptionen ist eine länderübergreifende Zusammenführung von Informationen aus Versorgung und Forschung unerlässlich. Um die notwendige Interoperabilität von Datensammlungen bzw. Registern zu erreichen, müssen Standards auf nationaler und internationaler Ebene geschaffen und eingeführt werden.

Spätestens seit den Empfehlungen durch das europäische Fachgremium EUCERD [3] zur „Registrierung und Datenerhebung von Patienten mit SE“ haben zahlreiche nationale und internationale Institutionen und Gruppen die EU-Empfehlungen aufgegriffen und 


\section{Positionspapier}

Tab. 1 Definitionen im Zusammenhang mit Registern von EUCERD bzw. aus der Literatur [10]

\section{Begriff} Erklärung

Register, Datensatz, Datensche-

ma

Metadaten

Verzeichnis von Registern Seltener Erkrankungen ter
„Ein Register ist eine möglichst aktive, standardisierte Dokumentation von Beobachtungseinheiten zu vorab festgelegten, aber im Zeitverlauf erweiterbaren Fragestellungen, für die ein präziser Bezug zur Zielpopulation transparent darstellbar ist" [11] „A patient registry is an organized system that uses observational study methods to collect uniform data (clinical and other) to evaluate specified outcomes for a population defined by a particular disease, condition, or exposure, and that serves one or more predetermined scientific, clinical, or policy purposes" [13]

Metadaten sind Daten über die Struktur von Daten. So kann bspw. ein gut dokumentiertes Datenschema die Bedeutung (Semantik) und die möglichen Ausprägungen (Wertebereich) von Datensätzen beschreiben. Metadaten sind zu unterscheiden von Auswertungen oder Aggregationen von Datensätzen bzw. Inhalten (Metainhalt), z. B. aggregierten Datensätzen aus einem Regis-

Ein Metadatenverzeichnis (Metadata Repository (MDR)) ist ein Register über Elemente aus Datenschemata. Diese werden wiederum strukturiert abgelegt. Das Datenschema eines MDR kann sich bspw. an den ISO/IEC-Standard 11179 anlehnen. Zweck ist, die Datenschemata - und in der Folge auch die Datensätze - zwischen unterschiedlichen Datensammlungen (Registern, Studien, Datenbanken etc.) vergleichbar zu machen

Ein „Verzeichnis von Registern“ soll die bereits bestehenden Register Seltener Erkrankungen für Patienten und Wissenschaftler öffentlich sichtbar machen. Im Unterschied zum Metaregister ist das Webportal im Internet frei einsehbar. Es arbeitet nicht auf Patienten- oder Fallebene, sondern beschreibt die angeschlossenen Register als Ganzes, vergleichbar mit dem Biobankenregister der TMF, das nicht auf Probenebene sammelt, sondern seine Datensätze aus Fragebögen gewinnt, die teilnehmende Biobanken ausfüllen
Strategien bzw. konkrete Empfehlungen für die Verbesserung oder den Aufbau von Patientenregistern zu SE publiziert. Außerdem beinhalten verschiedene nationale und europäische (Forschungs-)Projekte bereits das Thema Register, auch für Seltene Erkrankungen (s. - Infobox 1).

Darüber hinaus wurden aus dem Gesundheitsprogramm der EU-Kommission Mittel für den Aufbau einer europäischen Plattform für die Registrierung Seltener Krankheiten (European Rare Disease Registry Infrastructure, ERDRI) an der gemeinsamen Forschungsstelle der Europäischen Kommission (Joint Research Center (JRC), Ispra, Italien) zur Verfügung gestellt. Diese Plattform soll u.a. dazu dienen, einen zentralen Zugangspunkt $\mathrm{zu}$ Informationen im Bereich SE zu bieten, neue und vorhandene Register im Hinblick auf ihre Interoperabilität zu unterstützen und ITWerkzeuge zur Pflege erhobener Daten vorzusehen. Im Interesse einer europaweiten Vernetzung von Registern zu SE und auch von deutschen Registern sollte die Möglichkeit einer technischen Anbindung an die europäische Plattform möglich sein. Es wurde eine Untergruppe zur Beratung der europäischen Plattform für die Registrierung Seltener Krankheiten etabliert.

Unter Koordination des JRC wurde auf Basis des „EUCERD minimal data set" ein Minimaler Datensatz (MDS) ent- wickelt, der als Startbasis für eine gemeinsame Schnittmenge an Daten zwischen Registern in Europa dienen soll. Dieser MDS, der zwischen den Arbeitsgruppen abgestimmt wurde, die einen MDS für die Verwendung bei SE publiziert hatten (EUCERD, EPIRARE, RD-Connect), wurde veröffentlicht [9].

\section{Begriffsdefinitionen}

Im Rahmen der EUCERD-Registerempfehlungen [3] wurde eine Sammlung von Begriffen und Definitionen im Zusammenhang mit Registern zusammengetragen und veröffentlicht (s. [10]). Sie kann als „work in progress“ betrachtet und dem aktuellen Stand angepasst bzw. erweitert werden. In - Tab. 1 sind einige Definitionen aus der Literatur aufgeführt.

\section{Entwicklung und Betrieb eines Registers zu SE}

Register sind komplexe Vorhaben, bei denen die Dokumentation unterstützt wird durch eine personelle, technische und organisatorische Infrastruktur. Entsprechend umfassend sind Entwicklung, Umsetzung und Betrieb eines Registers $\mathrm{zu}$ organisieren. Insbesondere für die Entwicklung eines Registers empfiehlt das Deutsche Netzwerk Versorgungsforschung (DNVF) ein sogenanntes Registerprotokoll $[11,12]$. Vom DNVF wurden dazu die wichtigsten Aspek- te der Entwicklung in ihrer logischen und zeitlichen Abfolge benannt. Die für das jeweilige Vorhaben getroffenen Entscheidungen können in der Struktur des Registerprotokolls dokumentiert und nachverfolgt werden.

In Übereinstimmung mit den Empfehlungen der US-amerikanischen Agency for Healthcare Research and Quality (AHRQ; [13]) wird die Entwicklung in eine Phase der Planung mit groben Festlegungen und eine Phase des Entwurfs mit differenzierten Ausführungen unterteilt. Der nach Abschluss der Planungsphase formulierte Stand eignet sich als Grundlage für Projektanträge und Finanzierungsanfragen. Ähnliche Vorschläge wurden in der EU durch die Cross-border-Patient-Registries-Initiative (PARENT) unter besonderer Berücksichtigung einer grenzübergreifenden Nutzung erarbeitet [14]. Nachfolgend wird ein kurzer Überblick über die zu berücksichtigenden Aspekte in der Planungs-, Entwurfs- und Umsetzungsphase gegeben.

\section{Die Planungsphase}

- Tab. 2 gibt einen Überblick über die in der Planung zu bearbeitenden Aspekte.

\section{Die Entwurfsphase}

Nach Abschluss der Planungsphase, Klärung der Finanzierung sowie Entschei- 


\section{Tab. 2 Aspekte in der Planungsphase eines Registers}

\section{Fragestellungen und Aufgaben eines Registers}

Diese ergeben sich aus der Motivationslage der interessierten Personen und Gruppen sowie der Zielsetzung des Vorhabens. Die Konfiguration des Registers leitet sich dann wesentlich von den formulierten Fragestellungen ab. Die Fragestellungen sollten möglichst konkret benannt werden, auch wenn bei einem Register deren Fortschreibung im Betrieb typisch ist

\section{Datengrundlage}

Für jede einzelne Fragestellung sind die zur Beantwortung benötigten Merkmale zu identifizieren. Diese lassen sich zumeist den Dokumentationseinheiten Patient, Maßnahme, Ergebnis und Rahmenbedingungen zuordnen. Eine weitere Formalisierung ist an dieser Stelle nicht erforderlich

\section{Zielpopulation, Quellpopulation und Auswertungskollektiv}

Die verschiedenen Kollektive sind zu benennen sowie mit - ggf. geschätzten - Fallzahlen zu hinterlegen. Über die Zielpopulation soll später eine Aussage getroffen werden, sodass es sich hierbei typischerweise um alle Patienten mit einer bestimmten SE in Deutschland handelt. Aus der Quellpopulation werden die Probanden für das Register rekrutiert, z. B. aus den wesentlichen Zentren für die interessierende SE. Die Quellpopulation reduziert sich z. B. durch begrenzte Ressourcen, Ausschlusskriterien, Datenfehler oder fehlende Einwilligungen auf das Auswertungskollektiv

\section{Erhebungsverfahren}

Aus den Datengrundlagen, der Quellpopulation sowie den Rahmenbedingungen des Vorhabens leitet sich die Entscheidung über die anzusprechenden Erhebungszentren (z. B. Krankenhäuser), den Meldeweg (z. B. elektronisch über ein System zum Electronic Data Capture (EDC)), eine Nachverfolgung („Follow-up“) sowie die Zeitplanung (begrenzt, ohne festgelegtes Ende) ab. Neben der Zahl an Probanden und dem Umfang der Datenerhebung ist das Erhebungsverfahren für die Kosten des Registers leitend

\section{Trägerschaft und Verantwortlichkeiten}

Die Rechtsform des Registers ist ebenso wie dessen Finanzierung zu konzipieren. Für den Betrieb stehen neben einer zentralen Lösung verteilte Modelle zur Verfügung. Bei diesen können Aufgaben wie IT-Betrieb, Datenmanagement und Auswertung durch verschiedene Einrichtungen übernommen und über vertragliche Regelungen an die koordinierende Stelle angebunden werden. Letztere wird zumeist noch der inhaltlich verantwortlichen Stelle (wie einer Fachgesellschaft oder einer Patientenorganisation) zugeordnet sein. Datenschutzrechtlich wird in den allermeisten Fällen von einer Erfassung der Daten auf Grundlage einer Einwilligung und Probandeninformation auszugehen sein. Zur Haltung der personenbezogenen Daten ist dann eine Trennung der Patientenliste von den pseudonymisierten, inhaltlichen Daten vorzusehen, was bei der Festlegung von Verantwortlichkeiten zu berücksichtigen ist. Die Kontaktaufnahme mit einer Ethikkommission ergibt sich bei Registern - auch ohne diagnostische und therapeutische Intervention - aus Regelungen von Berufsordnungen oder beteiligten Einrichtungen wie Universitätskliniken. Zu klären sind auch der Verbleib der Daten nach Abschluss oder Einstellung des Registers sowie die Besitzverhältnisse im Betrieb

dung über eine Umsetzung erfolgt die Ausdifferenzierung der groben Überlegungen in der Entwurfsphase. - Tab. 3 gibt einen Überblick über die in der Entwurfsphase zu bearbeitenden Aspekte.

\section{Die Umsetzungsphase}

Bei der Umsetzung eines Registers sind nicht nur IT-Aspekte zu berücksichtigen. So gilt es bei eigenständiger Rechtsform, eine komplett neue Institution $\mathrm{zu}$ schaffen, mit Räumen, Personal, Einrichtung und Prozessen. Bezogen auf die IT sind Lösungen für verschiedene Ebenen umzusetzen. Für die Hardware der zentralen Anwendungen wie dem elektro- nischen Datenerfassungssystem (EDC) bietet sich ein Betrieb über IT-Dienstleister an. Zumindest für den Kernprozess des Datenmanagements findet sich inzwischen eine Reihe von kommerziellen und nichtkommerziellen Softwareprodukten [16]. Hierbei ist sowohl ein eigener Betrieb auf einer ggf. gemieteten Hardware denkbar als auch der Einkauf als Dienstleitung von dritter Stelle. Hardware und Software sind über ein professionelles IT-Servicemanagement abzusichern, welches z.B. über die Position eines leitenden Informationsbeauftragten (Chief Information Officer) des Registers organisiert wird. Im operativen Registerbetrieb werden dann Daten- management, Kommunikation mit den Studienzentren, Management der Probanden und Auswertung als Kernprozesse organisatorisch zusammengefasst. Die Gesamtverantwortung liegt bei der Geschäftsführung. Je größer ein Register aufgestellt ist, umso eher werden einzelne Aufgaben auf verschiedene Personen oder Einrichtungen aufgeteilt, für die dann auch Back-up-Strategien festzulegen sind. Bei sehr kleinen Vorhaben kann hingegen an eine Lösung über eine Stelle oder eine Delegation an einen Dienstleister gedacht werden, soweit dies nicht gegen datenschutzrechtlich relevante Forderungen verstößt.

Die Aufnahme des Registerbetriebs kann an der Rekrutierung des ersten Probanden und einer ersten Übermittlung von Daten festgemacht werden. Neben den sich dann anschließenden Routineaufgaben wird sich die Befassung mit den in diesem Kapitel beschriebenen Aspekten von Planung, Entwurf und Umsetzung bei sich fortentwickelnden Registern wiederholen, um die Konzeption immer wieder an neue oder sich verändernde Zielsetzungen, Aufgaben und Fragestellungen anzupassen.

\section{Softwaremodule der IT- Infrastruktur von SE-Registern}

$\mathrm{Zu}$ den Kernprozessen im Betrieb eines Registers zählen Datenmanagement, Analyse und Auswertung, Probandenmanagement und Kommunikation mit Studienzentren. Diese werden ergänzt durch die Prozesse der Entwicklung und ggf. des Abschlusses eines Registers sowie unterstützende Prozesse wie Personalverwaltung und IT-Servicemanagement. Einschlägige und zum Teil spezifische Softwareprodukte decken hiervon insbesondere das Datenmanagement ab [17]. Für Analyse und Auswertung werden hingegen häufig gängige Statistikpakete eingesetzt [18]. Im Bereich des Probandenmanagements und der Pseudonymisierung sind eigenständige Lösungen aus Projekten der vernetzten medizinischen Forschung entstanden. Bei der Kommunikation mit Studienzentren kann an die Nutzung von Software aus dem Umfeld des Kundenbeziehungsmanagements (Customer-Rela- 
Tab. 3 Aspekte in der Entwurfsphase eines Registers

\section{Datensatz}

Der Datensatz ergibt sich aus der in der Planungsphase identifizierten Datengrundlage. Er kann zuerst semiformal in einem Merkmalskatalog und dann als Ausgangspunkt für eine IT-technische Umsetzung in einem Informationsmodell festgehalten werden. Der Merkmalskatalog wird Anlage zum Registerprotokoll. Der Datensatz etablierter Register kann ebenso wie nationale und internationale Empfehlungen zur Prüfung von Vollständigkeit und Standardkompatibilität des Datensatzes genutzt werden

\section{Meldung und Erfassung}

Für jeden Meldezeitpunkt sind die zu übermittelnden Merkmale festzuhalten. Hieraus ergeben sich die Erhebungsformulare für den jeweiligen Meldezeitpunkt, die dann in der Umsetzungsphase elektronisch oder als Papierbogen implementiert werden. Auch eine an Ereignissen orientierte Erhebung ohne definierte Meldezeitpunkte sollte hier definiert werden. Wenn diese im Vordergrund steht, stellt dies besondere Bedingungen an ein ggf. einzusetzendes EDC-System. Für die im Regelfall erforderliche Pseudonymisierung ist das Verfahren organisatorisch und technisch zu definieren

\section{Datenmanagement}

Für ein zentrales Monitoring mit kontinuierlicher Überprüfung der erfassten Daten sowie einem Austausch mit den Studienzentren sind personelle Ressourcen vorzusehen. Die Möglichkeit eines Originaldatenabgleichs ist konzeptionell und organisatorisch zu prüfen. Falls dieser bei vorhandenen Originaldaten (z. B. Patientenakten) möglich ist, sollte seine Durchführung zumindest in einer Stichprobe der Meldungen erwogen werden. Zum Datenmanagement gehört eine regelmäßige Rückmeldung zur Datenqualität an die Studienzentren

\section{Datenschutz, Recht und Ethik}

Ein Datenschutzkonzept [15] ergänzt das Registerprotokoll und formuliert die datenschutzrechtlich relevanten Aspekte des angestrebten Registers aus. Registerprotokoll und Datenschutzkonzept stellen die wesentlichen Dokumente zur Begutachtung des Vorhabens durch betriebliche Datenschutzbeauftragte, Aufsichtsbehörden und Ethikkommissionen dar. Einwilligungserklärung und Probandeninformation sind Anlagen des Datenschutzkonzepts. Zur Gestaltung von Einwilligungserklärung und Probandeninformation finden sich Empfehlungen und unterstützende Werkzeuge bei der TMF - Technologie- und Methodenplattform für die vernetzte medizinische Forschung e. V. (s. http://www.tmf-ev.de/)

\section{Analyseplan}

Die zur Beantwortung der Fragestellungen durchzuführenden statistischen Analysen sind mit dem jeweiligen Zeitpunkt und den anzuwendenden Methoden zu beschreiben und sollten mit den Datensatzanforderungen bereits abgedeckt sein. Aus Gründen der Motivation sind Rückmeldungen an die Studienzentren in kürzeren Abständen vorzusehen („Berichtswesen“)

\section{Rekrutierung der Zentren}

Für die in der Planung beschriebenen Erhebungszentren sind die konkreten Einrichtungen zu identifizieren, anzusprechen und vertraglich einzubinden. Die Qualität der Meldungen ist stark von der Motivationslage abhängig. Anreizsystemen (finanziell, wissenschaftlich, Mehrwert etc.) kommt daher für den Erfolg des Registers eine besonders große Bedeutung zu. Bei finanziellen Anreizsystemen ist auf eine Kopplung mit der Vollständigkeit und Richtigkeit von Meldungen zu achten [15]

\section{Beratungsgremium und Lenkungsausschuss}

In komplexeren Vorhaben wird die Verantwortung für das taktische und strategische Management bei einem Lenkungsausschuss liegen. Die verschiedenen Stakeholder lassen sich über ein Beratungsgremium einbinden. Ein Ausschuss Datenschutz befasst sich mit datenschutzrechtlichen Fragen im Betrieb, z. B. mit der Zulässigkeit von Auswertungen für Dritte, falls diese z. B. zur Finanzierung vorgesehen sind. Das Verfahren bei wissenschaftlichen Publikationen könnte über eine Ordnung geregelt und durch ein gesondertes Gremium begleitet werden. Weitere Gremien ergeben sich nach der Zielsetzung und Ausgestaltung eines Registers

tionship-Management) gedacht werden [19]. Angesichts überschaubarer Zahlen von Probanden und Studienzentren bei Registern zu SE erscheint allerdings die Nutzung eigenständiger Module für das Probandenmanagement und für die Kommunikation mit Studienzentren entbehrlich. Bei der im Regelfall vorzunehmenden Trennung zwischen den identifizierenden und den pseudonymisierten, inhaltlichen Daten ist zusätzlich ein Modul zur Verwaltung von Patientenliste und Pseudonymen sowie unter Umständen ein weiteres Modul zur dop- pelten Pseudonymisierung erforderlich [20].

Im TMF-Projekt „Von der Evaluierung zur Konsolidierung: Anforderungen an Kohortenstudien \& Register IT“ (KoRegIT) wurden die Kernprozesse um eine Beschreibung von TopLevel-Aufgaben mit zugeordneten Anwendungsszenarien ergänzt [17]. Diese Differenzierung kann für eine Ausgestaltung der IT-Infrastruktur durch eine an spezifischen Anforderungen orientierte Auswahl eines Softwareprodukts herangezogen werden. Dem Kernprozess des Datenmanagements entsprechen dabei folgende Top-LevelAufgaben: Datenerhebung und Datenerfassung, Monitoring, Bereitstellung von probandenbezogenen Informationen, Datenmanagement (Organisation und Pflege der Daten), Studienunterstützung, Berichterstattung, Datenintegration und Datenzusammenführung sowie Unterstützung der Patientenversorgung. Insbesondere die Bereitstellung von probandenbezogenen Informationen, die Studienunterstützung sowie die Unterstützung der Patientenversorgung werden bislang nur von einem Teil der am Markt verfügbaren Softwareprodukte angeboten.

\section{Zuständigkeit bei der Erfassung von Daten}

Bei der Eingabe von Daten kann zwischen arztbasierten und patientenbasierten Registern unterschieden werden; bei arztbasierten Registern sollte die Eingabe von Daten nur durch Ärztinnen/ Ärzte oder Personal von Versorgungseinrichtungen für Menschen mit SE erfolgen. Jedoch ist auch die Eingabe durch Mitarbeiter von sozialpädiatrischen Zentren, Frühförderstellen, Kliniken mit entsprechender Spezialisierung o. Ä. denkbar. Im Regelfall muss die Einwilligung der Betroffenen und/oder der Erziehungs- und Sorgeberechtigten eingeholt werden. Alternativ kann die Datenerfassung durch die Patienten selbst (z.B. online via Webbrowser) erfolgen. Auch dann ist zur Verarbeitung im Regelfall eine informierte Einwilligung (,informed consent") einzuholen. In der Einwilligungserklärung stimmt 
der Patient beispielsweise der Weitergabe der nichtidentifizierenden Daten an eine europäische Datenbank zu und erklärt, ob er wünscht, Informationen zu krankheitsspezifischen Projekten oder zu klinischen Studien zu erhalten. Bei Registern, bei denen Patienten und Mitglieder des Behandlerteams gemeinsam Daten eingeben, ist die Markierung der Herkunft der Daten etabliert, falls Daten von beiden Quellen stammen können. Häufig ist aber der Bereich der vom $\mathrm{Pa}$ tienten erfassten Merkmale ein anderer als derjenige von Ärzten und anderen Behandlern.

\section{Pharmakovigilanz- und PROM- Module}

Innerhalb von arzt- und von patientenbasierten Registern ist es möglich, ein Pharmakovigilanzmodul zu integrieren, um die Sicherheit der Arzneimitteltherapie nach Zulassung über die Zeit zu beobachten. Insbesondere beineuen Arzneimitteln, für die noch keine Langzeitdaten verfügbar sind, stellt dies ein sinnvolles Vorgehen dar. Neben der Abfrage von Medikamenten, Dosierung, Einnahmeintervall und Nebenwirkungen können funktionelle Parameter, Skalen und Scores (sog. „patient reported outcomes measures“ (PROMs)) erhoben und mit den Daten zur Arzneimitteltherapie korreliert werden.

\section{Datenschutz bei Registern}

In Registern werden gesundheitsbezogene Daten erfasst, die häufig bereits an anderer Stelle erhoben und dokumentiert wurden, z. B. in der Akte eines Krankenhauses oder der Akte eines niedergelassenen Arztes. Teilweise werden für Register auch Daten neu erhoben, z. B. zur Lebensqualität. Im Regelfall handelt es sich um personenbezogene Daten, d.h. Daten, aus denen auch bei Wegfall identifizierender Merkmale wie Vorname, Nachname oder Geburtsdatum auf eine Person zurückgeschlossen werden kann. Die Daten werden zur Beantwortung von vorab formulierten Fragestellungen in der erforderlichen Detailtiefe erfasst oder lassen sich mit weiteren Angaben und Hintergrundwis- sen zusammenführen. Diese Situation macht eine datenschutzrechtliche und ethische Prüfung eines Registers erforderlich. Das Erfordernis einer datenschutzrechtlichen Prüfung ergibt sich aus den entsprechenden Gesetzen und Verordnungen (u.a. der Europäischen Union, der Bundesrepublik Deutschland oder der Bundesländer). Das Erfordernis einer ethischen Prüfung ergibt sich aus der Berufsordnung für Ärztinnen und Ärzte oder Regelungen beteiligter Einrichtungen wie Universitätskliniken. Falls im Rahmen eines Registers eine kontrollierte diagnostische, therapeutische oder sonstige Intervention erfolgt, können andere Regelungen wie das Arzneimittelgesetz relevant werden.

Die Erfassung von gesundheitsbezogenen Daten in einem Register setzt eine rechtliche Grundlage voraus. Liegt kein anderweitiger Erlaubnistatbestand vor, ist eine informierte Einwilligung der Teilnehmer zwingend notwendig. Dies ist im Regelfall die informierte Einwilligung der Patientinnen und Patienten, bei Minderjährigen der Erziehungsoder Sorgeberechtigten sowie bei Erwachsenen ggf. auch der rechtlichen Betreuer. Die inhaltlichen und formalen Anforderungen an eine solche rechtswirksame Einwilligung zur Verarbeitung personenbezogener Daten finden sich in den genannten Gesetzen. Die technisch-organisatorischen Anforderungen zum Schutz vor Risiken bei der Datenverarbeitung innerhalb eines Registers sind datenschutzrechtlich sowie informationstechnisch $\mathrm{zu}$ überprüfen und vor dem Start des Registers in einem Datenschutz- sowie Informationssicherheitskonzept schriftlich $\mathrm{zu}$ formulieren. Weiterhin fordert z. B. die DatenschutzGrundverordnung (DSGVO) explizit ein Verzeichnis aller Verarbeitungstätigkeiten.

Eine Beratung oder Bestätigung durch Datenschutzbeauftragte des Bundes oder der Länder ist im Regelfall nicht verpflichtend.

Die Registerleitung beantragt eine Beratung und Stellungnahme $\mathrm{zu}$ ethischen Gesichtspunkten. Beratung und Stellungnahme erfolgen entweder bei der Ethikkommission der eigenen Einrichtung, z.B. einer medizinischen Fa- kultät/einem Universitätsklinikum oder - falls eine Ethikkommission der eigenen Einrichtung nicht existiert - bei der Ethikkommission der zuständigen Landesärztekammer. Diese Verpflichtung gilt auch für Erhebungszentren; ggf. müssen also mehrere Ethikkommissionen einbezogen werden. Unterlagen für die ethische Beratung sind neben den Antragsformularen das Registerprotokoll, das Datenschutzkonzept sowie die Patienteninformation und die Einwilligungserklärung.

Auch bei informierter Einwilligung sind Grundsätze des Datenschutzrechts wie Datensparsamkeit und Zweckbindung zu beachten. Die Ausgestaltung der Einwilligungserklärung hat bei häufig langfristig angelegten Registern eine herausragende Bedeutung. Lücken in der Einwilligungserklärung sind später nur durch erneutes Ansprechen der Teilnehmer zu schließen, wobei das Ansprechen durch die verantwortliche Stelle bereits einen in der initialen Einwilligungserklärung zu berücksichtigenden Punkt darstellt. Die Zwecke, die das Register verfolgt, um seine Ziele zu erreichen, sind dabei so exakt wie möglich zu formulieren; die Datenerfassung beschränkt sich auf die Merkmale, die für die Zwecke notwendig sind.

Bei den vom Register benötigten Daten sind identifizierende Merkmale wie Vorname, Nachname und Geburtsdatum (IDAT) von den inhaltlichen Merkmalen wie Diagnose und Lebensqualität (MDAT) zu trennen. Damit verfügt ein Register typischerweise über 2 technisch und organisatorisch getrennte Datenbestände, einen der Vertrauens- oder Treuhandstelle (IDAT) und einen weiteren des Registerbetreibers (MDAT). Eine Pseudonymisierung der Daten sollte sicherstellen, dass eine Rückverfolgung der Datensätze zum Patienten nur in klar definierten Szenarien möglich ist.

Für Besonderheiten wie die Übernahme von Daten aus anderen Verfahren (z.B. der Abrechnung) oder die Feststellung des Verbleibs von Probanden über einen Abgleich der Teilnehmer mit Melderegistern ist in jedem Einzelfall eine Lösung zu entwickeln. Hierbei kann dann eine fachliche Beratung z.B. durch die Arbeitsgruppe Datenschutz der Techno- 
logie- und Methodenplattform für die vernetzte medizinische Forschung e.V. (TMF) empfohlen werden.

Die Teilnehmer haben umfassende Rechte zu Korrektur oder Widerruf ihrer Einwilligung wie zur Auskunft, Einsichtnahme, Korrektur oder Löschung ihrer Daten. Diese Szenarien müssen vorab geplant werden, um die erforderlichen Maßnahmen im Betrieb störungsfrei umzusetzen. $\mathrm{Zu}$ beachten ist, dass die Regelung in Ausnahmefällen, z. B. bei behördlichen Auflagen zur Sammlung sicherheitsrelevanter Daten, anders ausfallen kann. Bei größerer Zahl an Probanden ist dafür ein zentrales Einwilligungsmanagement sinnvoll, z. B. betrieben von der Vertrauens- oder Treuhandstelle.

\section{Empfehlung zum SE- Minimaldatenschema}

Als „SE-Minimaldatenschema“ (Minimal Data Set) oder SE-Basisdatensatz wird die Liste bezeichnet, welche die kleinste Menge gemeinsam benutzter Datenelemente definiert, d.h. die Liste der Datenelemente, die man ausnahmslos in allen SE-Registern finden sollte. Es gibt eine Reihe von publizierten konkreten Vorschlägen für die besondere Situation der Seltenen Erkrankungen [21, 22]. Aus diesen wurde eine praktikable Liste zur Verwendung in europäischen SE-Registern für Seltene Erkrankungen durch das JRC zusammengeführt, die lediglich 16 Datenelemente enthält [9].

\section{Langfristige Finanzierung/ Nachhaltigkeit}

Register steigen typischerweise im Wert, wenn sie über längere Zeiträume Daten erfassen, sofern Datenqualität und -menge nachhaltig gut sind. Hierzu ist eine gesicherte Finanzierung eine unerlässliche Voraussetzung. Dabei ist es grundsätzlich einfacher, Ärzte und Betroffene für einen begrenzten Zeitraum zu motivieren. Für eine über viele Jahre fortdauernde sorgfältige Datenerhebung sind zusätzliche Anreize zu schaffen. Eine finanzielle Absicherung der IT-Infrastruktur wird typischerweise in der Aufbauphase berücksichtigt, im Dauerbetrieb müssen diese
Kosten aber ebenso sichergestellt sein. Eine langfristige finanzielle Unterstützung bei der Motivation der Datenerfassung kann ebenso wichtig sein.

Einnahmen zur Unterhaltung einer generischen oder krankheitsspezifischen Registerinfrastruktur können durch eine Querfinanzierung aus anderen Bereichen angestrebt werden. Geeigneter ist jedoch eine die Unabhängigkeit des Registers sichernde eigenständige Finanzierung. Erstellung von Analysen im Auftrag Dritter sowie die Herausgabe von aggregierten Daten können zu Erlösen beitragen. Zusätzlich können Spenden, auch über einen Spendenbutton auf den angeschlossenen Webseiten genutzt werden. Ebenfalls besteht die Möglichkeit, dass die pharmazeutische Industrie Interesse an Daten zu bestimmten Seltenen Erkrankungen hat. Die Entscheidung über die Zulässigkeit einer Datenweitergabe gegen finanzielle Honorierung steht grundsätzlich unter dem Vorbehalt der informierten Einwilligung durch die Betroffenen. Nur wenn die Datenweitergabe Bestandteil der Patienteneinwilligung war, kann eine Auswertung bei einer konkreten Anfrage weitergegeben werden.

\section{Patientenorientierung}

Für die Patientinnen und Patienten mit einer Seltenen Erkrankung sind hochwertige medizinische Patientenregister sowohl in der Versorgungsforschung als auch in der Grundlagen- und der klinischen Forschung von hoher Bedeutung. Per Definition führt die geringe Anzahl von Patienten mit einer bestimmten Seltenen Erkrankung dazu, dass sowohl die Krankheitsentwicklung als auch die Wirkungen und Nebenwirkungen von möglichen Behandlungsansätzen über längere Zeit gesammelt und erforscht werden müssen, um dadurch eine verlässliche statistische Basis für die Beurteilung und Prognose zu erlangen, wie sie für häufigere Erkrankungen üblich ist. Damit können Register klinische Studien für Zulassungsverfahren ergänzen, dienen der Patientensicherheit und ermöglichen bei Seltenen Erkrankungen erst deren gezielte Erforschung.

Zum einen erwarten Patientinnen und Patienten mit Seltenen Erkrankun- gen Register, die auf dem Gebiet ihrer Erkrankungen die Forschung mit ihren Gesundheits- bzw. Krankheitsdaten ermöglichen. Für diesen Zweck willigen sie zu einem sehr hohen Anteil in die Nutzung ihrer Daten ein. Die Erwartung ist dabei, dass keine Datenhalden erzeugt werden, die am Ende wissenschaftlich nicht auswertbar und nutzbar sind, sondern Register, die sich an Standards und Best Practices orientieren, u. a. hinsichtlich der Interoperabilität. Dazu ist die Transparenz der Formulare und Merkmalskataloge (sog. Metadaten) sowohl bei klinischen Studien als auch den Registern eine wichtige Voraussetzung [23].

Zum anderen erwarten Patienten, dass diese Register nach Ende einer Förderoder Projektlaufzeit nicht wieder eingestellt werden, sondern dass ihre Daten dauerhaft genutzt werden. Viele wollen ihre Daten sogar über das eigene Leben hinaus der Forschung bereitstellen. Dazu gehört auch die Freiheit der Einwilligung oder des nachträglichen Widerspruchs mit einem einfachen Verfahren, selbst wenn der ärztliche Ansprechpartner vor Ort oder sogar die Trägerschaft eines Registers wechseln. Schutz der Daten vor Verlust, Diebstahl, Ausspähung, Veruntreuung oder Missbrauch sind wesentliche Elemente zur Vertrauensbildung genauso wie eine vertrauenswürdige Trägerschaft eines Registers.

Viele Patienten erwarten darüber hinaus, Informationen zu den Forschungsergebnissen zu erhalten, die mithilfe ihrer Daten gewonnen wurden. Um diese Erwartungen und die Erfahrungen der Patienten wirkungsvoll einzusetzen, wird die Beteiligung von Patientenvertreterinnen und Patientenvertretern in den Beiräten oder Lenkungsausschüssen (Steering Committees) eines Registers gefordert.

\section{Diskussion}

Die Notwendigkeit von Registern zu Seltenen Erkrankungen ist unumstritten. In Registern werden gesundheitsbezogene Daten erfasst, die bereits an anderer Stelle erhoben und dokumentiert wurden, z. B. in der Akte eines Krankenhauses oder der Akte eines niedergelassenen Arztes, bzw. die zu diesem Zweck neu erhoben 
werden, z. B. zur Lebensqualität. Im Regelfall handelt es sich um personenbezogene bzw. personenbeziehbare Daten, d. h. Daten, die sich auf eine Person beziehen lassen oder auf diese zurückschließen lassen. Die Daten werden zur Beantwortung von vorab formulierten Fragestellungen in hoher Detailtiefe erfasst oder lassen sich mit weiteren Angaben und Hintergrundwissen zusammenführen.

Der Aufbau eines Registers bedarf grundsätzlich einer verbindlichen, rechtlichen Voraussetzung. Ein entsprechendes Registerkonzept regelt die komplexen strukturellen und organisatorischen Vorgänge wie Meldung, Umfang und Aufbau des Registers, Datenübermittlung, Datenabgleich sowie Widerrufsrechte u. Ä. als auch die Informationssicherheit und technischen Voraussetzungen u.v.m. Die Verarbeitung von personenbezogenen Daten macht darüber hinaus eine umfassende Prüfung eines Registers vor der Implementierung erforderlich. Die Erfordernisse einer datenschutzrechtlichen Prüfung ergeben sich aus den entsprechenden Gesetzen und das Erfordernis einer ethischen Prüfung ergibt sich aus der Berufsordnung für Ärztinnen und Ärzte sowie den Regelungen zuständiger Einrichtungen wie Universitätskliniken (s. Abschnitt „Datenschutz bei Registern").

Grundsätzlich sind Anforderungen des Datenschutzes wie Datensparsamkeit und Zweckbindung zu beachten. Die Ausgestaltung der Patientenaufklärung und besonders der Einwilligungserklärung hat diesbezüglich bei den zumeist langfristig angelegten Registern eine herausragende Bedeutung. Die Zwecke, die das Register verfolgt, um seine Ziele zu erreichen, sind dabei so exakt wie möglich zu formulieren. Die Datenerfassung beschränkt sich auf die Merkmale, die für die Zwecke notwendig sind.

Bei Einhaltung der Regeln zur informationellen Selbstbestimmung und des Datenschutzes können mit Registern ohne zusätzliche Risiken für den $\mathrm{Pa}$ tienten wissenschaftliche Erkenntnisse gewonnen und beispielsweise Therapieentwicklungen beschleunigt werden. Neben dem Risiko eines Missbrauchs der Daten, das durch Einhaltung der Regeln des Datenschutzes durch Anonymisie- rung oder sichere Pseudonymisierung minimiert werden kann, sind wesentliche Gefahren die überflüssige Erhebung von Daten oder die mangelnde Verfügbarkeit der erhobenen Daten. Dem kann nur durch Transparenz und Kontrolle durch den Registerbetreiber wirksam begegnet werden.

In der wissenschaftlichen Literatur zum sinnvollen Umgang mit großen Datenmengen hat sich das Prinzip FAIR auch im Zusammenhang mit Registern in den letzten Jahren durchgesetzt [24]. FAIR steht für die Datenattribute Findable, Accessible, Interoperable und Reusable, also die Forderung, dass Registerdaten auffindbar, zugänglich, interoperabel und wiederverwendbar sein sollten. Nur dann erfüllen sie die Anforderungen an ethisch vertretbare Datensammlungen zum wissenschaftlichen Gebrauch. Diese Sichtweise ist weitgehend unbestritten, wenn auch damit nicht alle notwendigen Voraussetzungen für Register aufgelistet sind. Aus dem hier beschriebenen Text leiten die Autoren eher die Forderung ab, dass gute Register sich dadurch auszeichnen, dass sie neben der Einhaltung einer Vielzahl von Gütekriterien auch eine Erfüllung der FAIR-Bedingungen anstreben.

$\mathrm{Zu}$ den weiteren wichtigen Eigenschaften von Registern für Seltene Erkrankungen in dem Sinn dieses Textes gehört die Einordnung der Datensammlung in den internationalen Kontext. Es hat Sinn, die Daten in einer Form $\mathrm{zu}$ erfassen und verfügbar $\mathrm{zu}$ machen, die internationale Vergleiche oder $\mathrm{Zu}$ sammenführungen ermöglicht. Eine Übersicht über verfügbare Register verhindert die Erstellung immer wieder neuer, kleinteiliger Datensammlungen.

Es gibt eine unüberschaubar große Zahl kleiner und kleinster Sammlungen von Patientendaten, die durch ihre begrenzte Verfügbarkeit von minderem Wert sind. Durch Einholung einer entsprechenden Einwilligung zur weitergehenden Nutzung der Daten beim Patienten können mit modernen ITInstrumenten auch kleine und kleinste Sammlungen in größere Register integriert werden. Voraussetzung ist neben der spezifischen Patienteneinwilligung eine hinreichende Beschreibung der Da- ten (Metadaten) und eine ausreichende Datenqualität. Wesentliche Elemente einer übergeordneten Infrastruktur zur Sicherung der Interoperabilität sind eindeutige Definitionen der einzelnen zu erfassenden Datenelemente (Beschreibung in einem Metadatenregister) und eine Verknüpfung der Metadaten mit einem Register, in dem alle bekannten Register erfasst sind (Verzeichnis der Register). Mit solch einer Infrastruktur können alle verfügbaren Datensammlungen sichtbar und eine mehrfache Erhebung von Daten überflüssig gemacht werden. Neben einer krankheitsspezifischen Registerinfrastruktur zusammen mit einem Verzeichnis der Register und einem Metadatenregister auf nationaler Ebene kann eine solche umfassende Struktur auch auf europäischer Ebene oder international konzipiert werden, um auch bei sehr seltenen Erkrankungen $\mathrm{zu}$ aussagefähigen Zahlen zu kommen.

Die Maßnahmen im nationalen Aktionsplan in Bezug auf Register sind ein wichtiger Schritt zur Vernetzung und zur Herstellung einer Interoperabilität der Sammlungen von Patientendaten bei Seltenen Erkrankungen in Deutschland. Der nächste notwendige und ethisch unverzichtbare Schritt ist nun die europäische und internationale Vernetzung von SE-Registern, wobei durch die geleisteten Vorarbeiten die Voraussetzungen in Deutschland ausgesprochen gut sind, um bei Einhaltung der strengen europäischen Anforderungen an den Datenschutz $\mathrm{zu}$ einem weitreichenden Ergebnis zu kommen. Jede Ausweitung der Nutzung solcher Daten muss aber durch eine Einwilligung des Patienten abgesichert sein.

\section{Korrespondenzadresse}

\section{Dr. Holger Storf}

Medical Informatics Group (MIG), Universitätsklinikum Frankfurt

Haus 33C 2. OG, Theodor-Stern-Kai 7, 60590 Frankfurt am Main, Deutschland storf@med.uni-frankfurt.de

Mitglieder der NAMSE-Registerstrategiegruppe. E. Bruns-Philipps; M. Dietrich; K. Schmidt; B. Grimbacher; G. Kienle; S. Melin; B. Quadder; R. Röhrig; J. Stausberg; H. Storf; F. Ückert; T.O.F. Wagner; M. Walter; ehemalige Mitglieder: K. Burger; R. Dörner; A. Klucken; C. Steinmüller 
Funding. Open Access funding provided by Projekt DEAL.

\section{Einhaltung ethischer Richtlinien}

Interessenkonflikt. M.C. Walter: Beratertätigkeit und Teilnahme an Ad-hoc-AdBoards für die Firmen Avexis, Biogen, Novartis, Pharnext, PTC, Sarepta, Santhera, Roche, Ultragenyx, Wave. H. Storf, J. Stausberg, G. Kindle, B. Quadder, M. Schlangen, F. Ückert und T.O.F. Wagner geben an, dass kein Interessenkonflikt besteht.

Für diesen Beitrag wurden von den Autoren keine Studien an Menschen oder Tieren durchgeführt. Für die aufgeführten Studien gelten die jeweils dort angegebenen ethischen Richtlinien.

Open Access. Dieser Artikel wird unter der Creative Commons Namensnennung 4.0 International Lizenz veröffentlicht, welche die Nutzung, Vervielfältigung, Bearbeitung, Verbreitung und Wiedergabe in jeglichem Medium und Format erlaubt, sofern Sie den/die ursprünglichen Autor(en) und die Quelle ordnungsgemäß nennen, einen Link zur Creative Commons Lizenz beifügen und angeben, ob Änderungen vorgenommen wurden.

Die in diesem Artikel enthaltenen Bilder und sonstiges Drittmaterial unterliegen ebenfalls der genannten Creative Commons Lizenz, sofern sich aus der Abbildungslegende nichts anderes ergibt. Sofern das betreffende Material nicht unter der genannten Creative Commons Lizenz steht und die betreffende Handlung nicht nach gesetzlichen Vorschriften erlaubt ist, ist für die oben aufgeführten Weiterverwendungen des Materials die Einwilligung des jeweiligen Rechteinhabers einzuholen.

Weitere Details zur Lizenz entnehmen Sie bitte der Lizenzinformation auf http://creativecommons.org/ licenses/by/4.0/deed.de.

\section{Literatur}

1. Nationaler Aktionsplan für Menschen mit Seltenen Erkrankungen (2019) Handlungsfelder, Empfehlungen und Maßnahmenvorschläge. https://www. namse.de/fileadmin/user_upload/downloads/ Nationaler_Aktionsplan.pdf. Zugegriffen:21. Apr. 2020

2. European Platform on Rare Diseases Registration (EU RD Platform, ERDRI) (2020) https://eu-rdplatform.jrc.ec.europa.eu/. Zugegriffen: 19. Jan. 2020

3. EUCERD Core Recommendations on Rare Disease Patient Registration and Data Collection to the European Commission (2013) Member states and all stakeholders. http://www.eucerd. eu/wp-content/uploads/2013/06/EUCERD Recommendations_RDRegistryDataCollection_ adopted.pdf.Zugegriffen: 19. Jan. 2020

4. Lablans M, Kadioglu D, Mate S, Leb I, Prokosch HU, Ückert F (2016) Strategien zur Vernetzung von Biobanken-Klassifizierung verschiedener Ansätze zur Probensuche und Ausblick auf die Zukunft in der BBMRI-ERIC. Bundesgesundheitsblatt Gesundheitsforschung Gesundheitsschutz 59:373

5. Muscholl M, Kadioglu D, Lablans M et al (2016) OSSE - Open-Source-Registersystem für Seltene
Erkrankungen in der EU. Version 1.0 vom 26.04.2016. https://www.osse-register.de/OSSE summary de.pdf.Zugegriffen: 30. Juli 2018

6. Universitätsmedizin Mainz (2017) Die Mainzelliste als OpenSource. www.mainzelliste.de. Zugegriffen:25. Aug. 2017

7. Storf H, Schaaf J, Kadioglu D, Göbel J, Wagner TOF, Ückert F (2017) Registries for rare diseases: OSSE-An open-source framework for technical implementation. Bundesgesundheitsblatt Gesundheitsforschung Gesundheitsschutz 60(5):523-531. https://doi.org/10.1007/s00103017-2536-7

8. EPIRARE (2014) The current situation, the challenges and the expectations on Patient registries and Databases. III Activities and needs of existing rare disease registries (Deliverable 1.3.)

9. European Platform on Rare Diseases Registration (EU RD Platform, ERDRI) Set of common data elements for rare diseases registration. https:// eu-rd-platform.jrc.ec.europa.eu/set-of-commondata-elements. Zugegriffen: 19. Jan. 2020

10. EUCERD (Hrsg) (2015) Thesaurus of registry terminology, EUCERD joint action WP8 task 8.1: integration of registry activities. http://www. eucerd.eu/wp-content/uploads/2015/03/WP8_ Registries_Thesaurus.pdf. Zugegriffen: 19. Jan. 2020

11. Deutsches Netzwerk Versorgungsforschung e.V. (DNVF) (2020) http://www.netzwerkversorgungsforschung.de. Zugegriffen: 23. Apr. 2020

12. Müller D, Augustin M, Banik N et al (2010) Memorandum Register für die Versorgungsforschung. Gesundheitswesen 72:824-839

13. Gliklich R, Dreyer N, Leavy M (Hrsg) (2014) Registries for evaluating patient outcomes: a user's guide, 3. Aufl. AHRQ publication no. 13(14)EHC111, Bd.2. Agency for Healthcare Research and Quality, Rockville

14. Zaletel M, Kralj M (Hrsg) (2015) Methodological guidelines and recommendations for efficient and rational governance of patient registries. National Institute of Public Health, Slovenia, Ljubljana

15. Nonnemacher M, Nasseh D, Stausberg J (2014) Datenqualität in der medizinischen Forschung. Leitlinie zum adaptiven Management von Datenqualität in Kohortenstudien und Registern, 2. Aufl. Medizinisch Wissenschaftliche Verlagsgesellschaft, Berlin

16. Stausberg J, Altmann U (2016) Register und Kohorten (2016. In: Drepper J, Semler SC (Hrsg) IT-Infrastrukturen in der patientenorientierten Forschung. Aktueller Stand und Handlungsbedarf. Verfasst und vorgelegt vom IT-Reviewing-Board derTMF. AKA, Berlin, S47-78

17. Michalik C, Dreß J, Ngouongo S et al (2014) Requirements and tasks of cohorts and registers, the German KoRegIT project. In: Lovis C, Séroussi B, Hasman A, Pape-Haugaard L, Andersen SK (Hrsg) e-Health-For Continuity of Care Proceedings of MIE 2014. IOS Press, Amsterdam, S 1085-1089

18. Stausberg J, Altmann U, Antony G, Drepper J, Sax U, Schütt A (2010) Registers for networked medical research in Germany. Situation and prospects. Appl Clin Inform 1:408-418

19. Schwanke J, Rienhoff O, Schulze TG, Nussbeck SY (2013) Suitability of customer relationship management systems for the management of study participants in biomedical research. Methods Inf Med 52:340-350

20. Pommerening K, Drepper J, Helbing K, Ganslandt T (2014) Leitfaden zum Datenschutz in medizinischen Forschungsprojekten - Generische
Lösungen derTMF 2.0. Medizinisch Wissenschaftliche Verlagsgesellschaft, Berlin

21. Choquet R, Maaroufi M, de Carrara A, Messiaen C, Luigi E, Landais P (2015) A methodology for a minimum data set for rare diseases to support national centers of excellence for healthcare and research. Journal of the American Medical Informatics Association 2014. Rubinstein YR, Mclnnes P. NIH/NCATS/GRDR ${ }^{\circledR}$ Common Data Elements: A leading force for standardized data collection. Contemp Clin Trials 42:78-80

22. Taruscio D, Mollo E, Gainotti S, Posada de la Paz M, Bianchi F, Vittozzi L (2014) The EPIRARE proposal of a set of indicators and common data elements for the European platform for rare disease registration. Archives of Public Health. 2014;72:35. doi:10.1186/2049-3258-72-35.EUCERD:Minimum Data Set for Rare Disease Registries. http:// www.eucerd.eu/wp-content/uploads/2015/03/ WP8_Registries_MDS.pdf RD Connect: https:// rd-connect.eu/what-we-do/phenotypic-data/ registry-common-data-elements/. Zugegriffen: 19. Jan. 2020

23. Dugas M, Jöckel KH, Gefeller O et al (2014) Freier Zugang zu Dokumentationsformularen und Merkmalskatalogen im Gesundheitswesen. Memorandum „Open Metadata“. GMS Med Inform Biom Epidemiol 10(1):Doc1. https://doi.org/10. 3205/mibe000150

24. Wilkinson MD, Dumomentier M, Aalbersberg IJ et al (2016) The FAIR guiding principlesfor scientific data management and stewardship, scientific data 3. Macmillan, New York https://doi.org/10.1038/ sdata.2016.18 